\title{
Our Experience in 33 Patients of Multiple Ventricular Septal Defect Closure
}

\author{
Periyasamy Thangavel, Ganapathy Sambandam Kamalakkannan, \\ Baskar Ranjith Karthekeyan*, Siva Muthukumar, Mahesh Vakamudi, Jebaraj Rethinasamy \\ Professor of Cardiothoracic and Vascular Surgery, Sri Ramachandra University, Chennai, India \\ Email: drsamy79@yahoo.com, anaeskamal2006@gmail.com, ${ }^{*}$ ranjithb73@gmail.com, drsivam@hotmail.com, \\ vakamudi@gmail.com, jebaraj66@gmail.com,
}

Received 25 September 2015; accepted 18 October 2015; published 21 October 2015

Copyright (C) 2015 by authors and Scientific Research Publishing Inc.

This work is licensed under the Creative Commons Attribution International License (CC BY). http://creativecommons.org/licenses/by/4.0/

(c) (i) Open Access

\section{Abstract}

Study: Retrospective study of 33 patients with multiple VSD during March 2009 to March 2014. Technique: The defects were located by injecting the cardioplegia solution into left atrium after occluding the pulmonary artery. Through right atrial approach, the large ventricular septal defects were closed by pericardial patch. The smaller ventricular septal defects were closed directly. Results: Out of 33 patients, 20 patients were multiple ventricular septal defects, 5 patients were atrioventricular canal defect with multiple ventricular septal defects and 8 patients were transposition of great arteries with multiple ventricular septal defects. All the patients had severe pulmonary arterial hypertension. The mean age and body weight at repair was 4.51 months and $5.41 \mathrm{~kg}$ respectively. Failure to locate additional ventricular septal defects happened in 1 patient where the pulmonary artery banding was done. Six patients had residual ventricular septal defect after surgery, and no significant left to right shunted. One patient had permanent pacemaker implanted in the postoperative period due to the heart block. There was no early and late mortality. Conclusion: Management of multiple ventricular septal defects is quite complex. Right atrial approach of ventricular septal defects closure is safe, simple and effective in closure of multiple ventricular septal defects.

\section{Keywords}

Ventricular Septal Defect, Pulmonary Arterial Pressure, Congenital, Infants

\section{Introduction}

The management of patients with multiple ventricular septal defects by primary repair is a surgical challenge

\footnotetext{
"Corresponding author.
}

How to cite this paper: Thangavel, P., Kamalakkannan, G.S., Karthekeyan, B.R., Muthukumar, S., Vakamudi, M. and Rethinasamy, J. (2015) Our Experience in 33 Patients of Multiple Ventricular Septal Defect Closure. World Journal of Cardiovascular Surgery, 5, 103-107. http://dx.doi.org/10.4236/wjcs.2015.510016 
and it is more demanding when it is neonates and infants. Perioperative identification of ventricular septal defects is difficult through the right atriotomy because of trabeculations. Closure of multiple ventricular septal defects requires long cardiopulmonary bypass and cross clamp time. Ventriculotomies might be required to access the multiple ventricular septal defects. Alternative techniques like pulmonary artery banding and device closure has its own technical limitations. We report our experience of closure of multiple ventricular septal defects located by injecting the cardioplegia solution into left atrium after occluding the pulmonary artery. Through right atrial approach, the large ventricular septal defects were closed by pericardial patch. The smaller ventricular septal defects were closed directly. This is a simple and effective approach without ventriculotomy.

\section{Materials and Methods}

33 patients were operated for multiple ventricular septal defects during March 2009 to March 2014. Out of 33 patients, 20 patients were multiple ventricular septal defects (Figure 1), 5 patients were atrioventricular canal defect with multiple ventricular septal defect and 8 patients were Transposition of great arteries with multiple ventricular septal defects. The mean age and body weight at repair was 4.51 months and $5.41 \mathrm{~kg}$ respectively. All the patients had severe pulmonary arterial hypertension. Failure to locate additional ventricular septal defects happened in 1 patient where pulmonary artery banding was done. Six patients had residual ventricular septal defect after surgery but no significant left to right shunt. One patient had permanent pacemaker implanted in the postoperative period due to heart block. There was no early and late mortality.

\section{Technique}

We use the right atrial approach for ventricular septal defect closure after aorto-bicaval cannulation and aortic cross clamp with antegrade cold blood cardioplegia. Right superior pulmonary vein left atrial vent was used. Through right atrial approach large ventricular septal defect closed by pericardial patch. Right atrium was opened and the tricuspid valve leaflets were retracted to expose the ventricular septal defect and the important surrounding structures (Figure 2). Double-armed sutures (5 - 0 or 6 - 0 ) with buttressing pledgets are placed 2 $3 \mathrm{~mm}$ away from the edge of the defect on the right ventricular side. This provides a wider contact area between the patch and the myocardium, helping avoid injury to the conduction bundle that often runs near the crest of the defect. The pledgets tend to bolster the attachment to the often-friable endomyocardium that surrounds the ventricular septal defect. In addition, we use gentle traction on the previously placed pledgetted suture to retract the adjacent proposed suture site into the field for accurate placement; this is particularly helpful when the ventricular septal defect extends to the aortic annulus. The smaller defects were located by injecting the cardioplegia solution into left atrium after occluding the pulmonary artery. The smaller ventricular septal defects were closed directly with pledgetted 5.0 prolene suture. Through the tricuspid valve, trabeculation was separated to find the mouth of ventricular septal defect. None of patient had ventriculotomy. One patient underwent PA band as we could not locate the ventricular septal defect. All patients underwent conventional ultrafiltation and modified

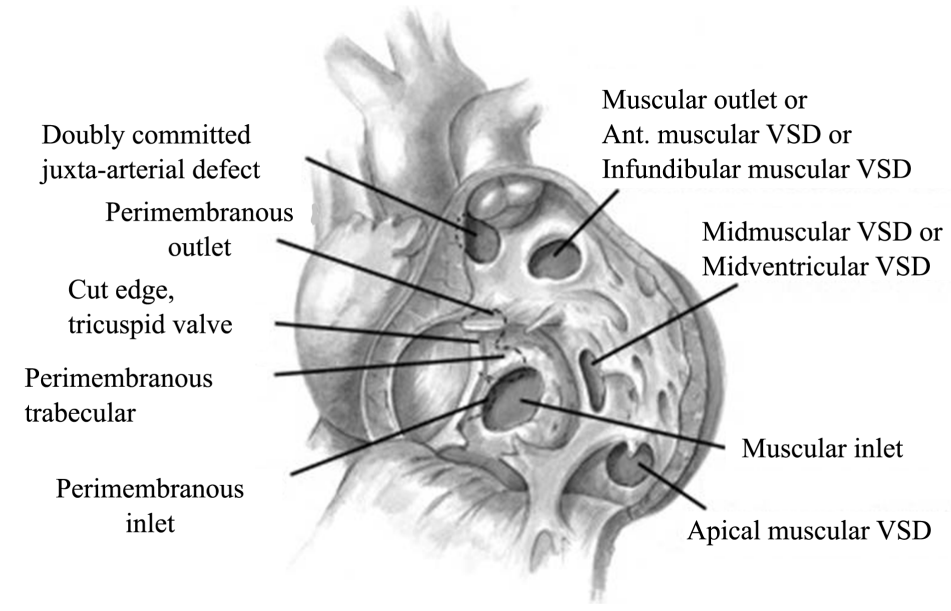

Figure 1. Diagram showing multiple VSD. 


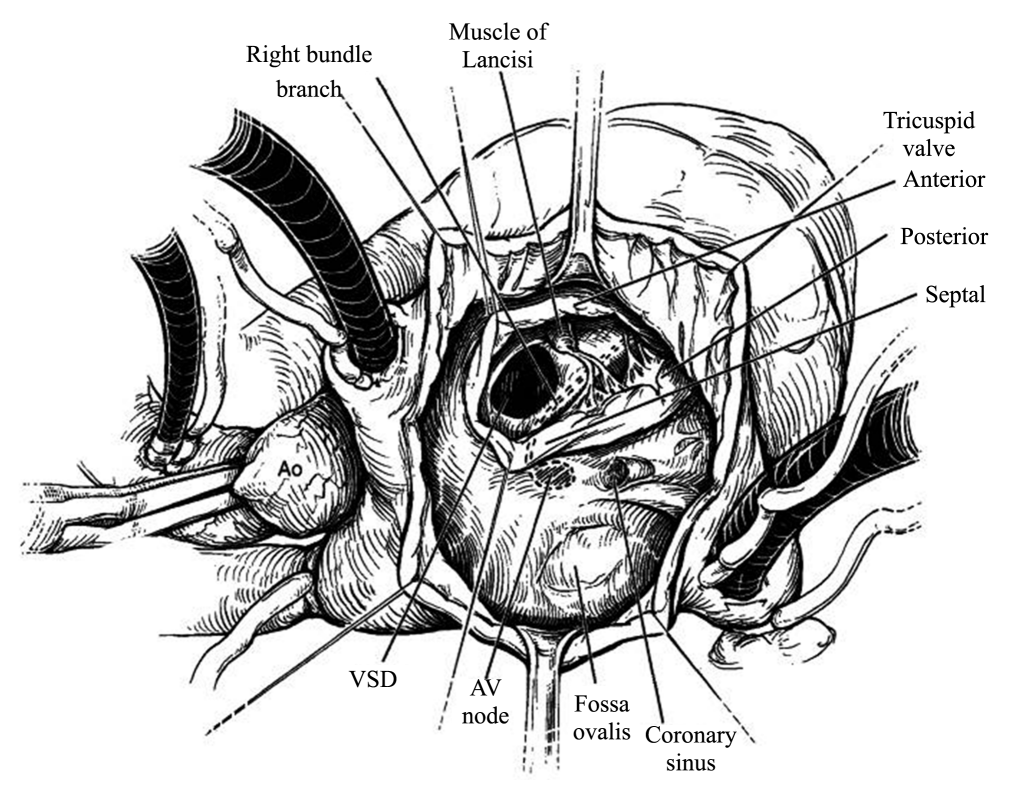

Figure 2. Right atrial approach for multiple VSD.

ultrafiltration. Completeness of ventricular septal defect closure was assessed with epicardial echocardiogram and if residual shunt present and blood samples were taken from the right atrium and pulmonary artery to calculate the magnitude of shunt. Transthoracic echocardiogram study was performed before discharge.

\section{Results}

5 patients had AV canal defect with multiple ventricular septal defect.8 patients had TGA with multiple ventricular septal defect. 20 patients had multiple ventricular septal defects. The mean age is 4.51 months. The mean weight is $5.41 \mathrm{~kg}$. There were 20 males and 13 females. The mean aortic cross clamp time was 86.6 minutes. The mean CPB time was 110.09 minutes. All patients had severe PAH. One patient had additional PA banding. One patient had permanent pacemaker implanted due to AV block. 6 patients had residual ventricular septal defect (but not significant L-R shunt). Post echocardiogram revealed RV dysfunction in 3 patients, LV dysfunction in 3 patients, mild PAH for 30 patients and moderate PAH for 3 patients. There were no in hospital deaths.

\section{Discussion}

Right atrial approach is the most frequently used approach and is usually applicable to the perimembranous, inlet, muscular and the left ventricle-to-right atrium types of ventricular septal defect. Closure of trabecular ventricular septal defect is usually first attempted through the right atrium, but sometimes the apical and the "Swiss cheese" subtypes require a limited right or left apical ventricular incision. With conventional techniques, VSDs can be difficult or impossible to identify intraoperatively in neonates and young infants. Through the tricuspid valve, in the search for the VSD, extensive division of muscular trabeculations and bands, including the moderator band, may be performed. These divisions compromise RV function substantially. A small apical RV incision, precisely targeted, can be valuable. This emphasizes the necessity to know exactly where the ventricular septal defects are. Alternately, several incisions in the quest of the ventricular septal defect, together with their closure, if extensive or ill placed, could cause considerable damage. The surgical exposition gained through left ventriculotomies can be disappointing. The leftward aspect of the septum is not free of trabeculations and may be heavily trabeculated at the apex. Moreover, left ventriculotomies are considered by some to be associated with unacceptable long-term risks, including aneurysm formation, LV dysfunction, and ventricular arrhythmia [1] [2].

Catheter based interventions are usually not recommended in this group of patients. The stiffness of the guide wire but especially of the sheath is responsible for rupture of the mitral valve, tearing of the septum, severe dysrhythmia, and cardiac arrest during the procedure. Initial palliation with a pulmonary artery band has inherent 
morbidity and mortality and requires two stages. The added complexity during the second stage includes pericardial adhesions, need for pulmonary artery repair, and RV hypertrophy contributing to the difficult location and closure of the ventricular septal defect [3]-[6].

Often, undiagnosed additional small ventricular septal defect without detectable shunting in the presence of a larger ventricular septal defect may be unmasked after repair and contribute to a significant residual left-to-right shunt. The technique presented here deals with these issues.

In our series, the technical difficulties were:

1. Without ventriculotomy and cutting trabeculations the location of VSD was difficult;

2. Some small ventricular septal defects are only visible only after closure of the other ventricular septal defect and may become hemodynamically significant;

3. Cross clamp time have been long, but these were in relation to complexity associated with the lesions.

Despite these practical limitations, the technique was used for all 33 patients. 13 patients underwent simultaneous complex cardiac repair. No ventriculotomy was used. One patient had additional PA band for inability to locate target ventricular septal defect. There was evidence of residual left-to-right shunting detected at postoperative studies in 6 patients. These shunts were not considered of significant magnitude.

Many surgical techniques were used for surgical closure of multiple ventricular septal defects. Kitagawa and colleagues [3] [4] used different techniques in 33 patients, one of which relies on the concept of an oversized patch placed on the LV side of the septum. Sutures were placed circumferentially through the patch and the rim of the ventricular septal defect from the LV side. Obviously, this method requires adequate localization and visualization of the ventricular septal defect during aortic cross-clamping and numerous sutures to be brought out from the LV, properly organized and tied. Five patients (15\%) required reinterventions for residual VSD; early mortality was 3\%. In 29\%, a left ventriculotomy was used, without, however, evidence of LV dysfunction at midterm follow-up. Kapoor and associates [7] used a simplified sandwich patch closure in 5 patients. They used a right-an-gled instrument to probe the septum from the LV side, depending on the presence of a sizeable perimembranous ventricular septal defect for introduction of the instrument. No follow-up data were given, and the patients were significantly older. Recently, Ootaki and collaborators [8] presented a series of 11 patients treated with a similar technique, sandwiching the septum between two felt patches. A total of 25 ventricular septal defects were present, and 15 were closed with the sandwich technique. The mean age was greater than in our series, and 9 of the 11 had primary palliation. Morbidity was not reported. Their report adds credit to the safety of the method. For both of these reports, however, the main difference from the technique described in this report is the absence of epicardial echocardiography and means of precise location of the ventricular septal defect before ischemic arrest. Probing the septum with instruments, especially in neonates' and infants' hearts, can be misleading and create communications when the ventricular septal defects do not exist. Mace and colleagues [9] as well as Black and coworkers [10] obliterate the ventricular septal defect by extensive patching of the RV side of the septum with a single or several patches. Operative and follow-up mortalities were zero in both series, and no reinterventions for residual shunts were required. Neither group reported experience with their technique in neonates. Mace found septal motional abnormalities in all patients. In that respect, we believe that our approach with one or more separate patch rather than one very large patch preserves the function of the septum better.

Larger studies focusing on outcome [2] [3] have reported reoperations for residual ventricular septal defect in $6 \%$ to $13 \%$, and in one series there was a $7.7 \%$ 30-day mortality [5]. Importantly, several studies emphasize the merits of primary surgical repair [2]-[4]. In our series there was no in hospital mortality. However we had RV dysfunction in 3 patients and LV dysfunction in 3 patients.

\section{Conclusion}

We believe this technique is safe, effective, simple and reproducible in dealing with multiple ventricular septal defects in infants. This technique may be valuable in complex ventricular septal defect aiming for primary repair.

\section{References}

[1] Hanna, B., Colan, S.D., Bridges, N.D., Mayer, J.E. and Castaneda, A. (1991) Clinical and Myocardial Status after Left Ventriculotomy for Ventricular Septal Defect Closure. Journal of the American College of Cardiology, 17, 110A. http://dx.doi.org/10.1016/0735-1097(91)91406-5 
[2] Wollenek, G., Wyse, R., Sullivan, I., Elliott, M., de Leval, M. and Stark, J. (1996) Closure of Muscular Ventricular Septal Defects through a Left Ventriculotomy. European Journal Cardio-Thoracic Surgery, 10, 595-598. http://dx.doi.org/10.1016/S1010-7940(96)80371-X

[3] Kitagawa, T., Kitaichi, T., Sugano, M. and Kurobe, H. (2013) Techniques and Results in the Management of Multiple Ventricular Septal Defects. General Thoracic and Cardiovascular Surgery, 61, 367-375. http://dx.doi.org/10.1007/s11748-013-0267-8

[4] Kitagawa, T., Durham, L.A., Mosca, R.S. and Bove, E.L. (1998) Techniques and Results in the Management of Multiple Ventricular Septal Defects. The Journal of Thoracic and Cardiovascular Surgery, 115, 848-856. http://dx.doi.org/10.1016/S0022-5223(98)70366-6

[5] Serraf, A., Lavour-Gayet, F., Bruniaux, J., Quaknine, R., et al. (1992) Surgical Management of Isolated Multiple Ventricular Septal Defects: Logical Approach in 130 Cases. The Journal of Thoracic and Cardiovascular Surgery, 103, 437-443.

[6] Seddio, F., Reddy, M.V., McElhinney, D.B., Tworetzky, W., Silverman, N.H. and Hanley, F.L. (1999) Multiple Ventricular Septal Defects (How and When Should They Be Repaired). The Journal of Thoracic and Cardiovascular Surgery, 117, 134-140.

[7] Kapoor, L., Gan Das, M.B., Mukhopadhyay, S. and Bandhopadhyay, A.A. (1999) Technique to Repair Multiple Muscular Ventricular Septal Defects. The Journal of Thoracic and Cardiovascular Surgery, 117, 402-403. http://dx.doi.org/10.1016/S0022-5223(99)70445-9

[8] Ootaki, Y., Yamaguchi, M., Yoshimura, N., Oka, S., Yoshida, M. and Hasegawa, T. (2003) Surgical Management of Trabecular Ventricular Septal Defects (the Sandwich Technique). The Journal of Thoracic and Cardiovascular Surgery, 125, 508-512. http://dx.doi.org/10.1067/mtc.2003.56

[9] Mace, L., Dervanian, P., Le Bret, E., Folliguet, T.A., Lambert, V., Losay, J., et al. (1999) “Swiss cheese” Septal Defects (Surgical Closure Using a Single Patch with Intermediate Fixings). The Annals of Thoracic Surgery, 67, 17541759. http://dx.doi.org/10.1016/S0003-4975(99)00325-2

[10] Black, M.D., Shukla, V., Rao, V., Smallhorn, J.F. and Freedom, R.M. (2000) Repair of Isolated Multiple Muscular Ventricular Septal Defects (the Septal Obliteration Technique). The Annals of Thoracic Surgery, 70, 106-110. http://dx.doi.org/10.1016/S0003-4975(00)01372-2 